\section{From science fiction to reality?}

SIR - The existence of a planet in orbit around a neutron star, reported by Bailes et al. ${ }^{1}$, was actually predicted ${ }^{2,3}$ by the science-fiction writer Poul Anderson in a novel first published in 1958. Anderson also predicted that a neutron star would possess "The most powerful bloody magnetic field ever noticed around any heavenly body" (ref. 3, p. 78 ), though his estimate of $10^{4}$ gauss for the surface field strength was much too low ( $10^{12}$ gauss for most neutron stars). Anderson argued that a neutron star must have a strong magnetic field, because the field strength should be proportional to the neutron star angular velocity, which should be enormous due to conservation of angular momentum during collapse of a stellar core. Anderson's physics is essentially correct. (Conservation of flux and angular momentum imply the proportionality of magnetic field strength and angular velocity.) Anderson's analysis was in advance of astronomical opinion. In a Science Fact article $^{4}$ (in the same issue of Astounding Science Fiction as Anderson's novel), the astronomer Alastair Cameron wrote "the remnant left behind [a supernova explosion] is a white dwarf star."

As proposed by Anderson, the neutron star's planetary companion would have "a smaller mass and radius than Earth" (ref. 3, p. 143), whereas the planet discovered by Bailes et al. has a most probable mass of about $10 M_{\mathrm{E}}$, where $M_{\mathrm{E}}$ is the mass of the Earth. Anderson makes the interesting suggestion that the planetary progenitor actually had a "mass greater than Jupiter" and "an extremely big orbit" but had its hydrogen atmosphere blown away by the supernova explosion (leaving only the nickel-iron core). The planet lost angular momentum in the process and its orbital radius became much smaller. Such a mechanism for generating an approximately Earth-mass planet does not seem to have been considered by Bailes et al . Angular momentum being carried away by the blown-off atmosphere could solve the binding energy problem ${ }^{1,5}$ : unless a planet loses angular momentum, it will become unbound if its star loses more than half its mass in less than one orbital period.

Bailes et al. point out that even if a mechanism could be found to keep a planet bound during a supernova explo-

\section{Scientific Correspondence}

Scientific Correspondence is intended to provide a forum in which readers may raise points of a scientific character. They need not arise out of anything published in Nature. In any case, priority will ge given to letters of fewer than 500 words and five references. sion, one would expect the planet's orbital eccentricity to be large, whereas the planet they discovered has an almost circular orbit. Anderson does not discuss this eccentricity problem, though he does mention two physical processes that might be relevant. First, he notes that once the outer layers of hydrogen are removed, the core would "revert to normal density, which would be a pretty spectacular catastrophe in itself" (ref. 3, p. 143). Indeed it would. Hubbard et al. ${ }^{6}$ have constructed models for the interiors of Jupiter and Saturn assuming both have a core mass of about $20 M_{\mathrm{E}}$. Thus an estimate of the energy released by blowing off the outer atmosphere of Jupiter and reducing its mass to Saturn's is $P_{\mathrm{J}} V_{\mathrm{J}}-P_{\mathrm{S}} V_{\mathrm{S}}$, where $V_{\mathrm{J}}$ and $V_{\mathrm{S}}$ are respectively the volumes of the cores of Jupiter and Saturn, and $P_{\mathrm{J}}$ and $P_{\mathrm{S}}$ are respectively the average pressures in the cores, obtained by a linear interpolation between the pressures at the centre and the surface of the cores, given in Table 4 of ref. 6 . The energy released is $3 \times 10^{41} \mathrm{erg}$, which is enough to accelerate about $7 M_{\mathrm{E}}$ ( 7 per cent of Saturn's total mass) to Saturn's escape velocity.

The supernova ejecta would be expected to blow back gas emitted toward the star, so the net impulse from this density change would be toward the star. But the most efficient way to reduce eccentricity is via an impulse directed tangential to the orbit (ref. 7, p. 349) at apastron. Friction between residual supernova eject and the planet was Anderson's mechanism of reducing the semimajor axis. This would also reduce eccentricity ${ }^{7}$, but it seems by an insufficient amount. It is nevertheless suggestive that the mass of the Bailes et al. planet has a mass roughly equal to the core masses of Jupiter and Saturn ${ }^{6}$.

Do these correct but qualitative predictions entitle Poul Anderson to any scientific credit? Perhaps pulsars with planets should be called 'Poulsars'.

\section{Department of Mathematics and} Department of Physics,

\section{Tulane University,}

New Orleans,

Louisiana 70118, USA

1. Bailes, M., Lyne, A. G. \& Shemar, S. L. Nature 352 311-313 (1991)

2. Anderson, P. We Have Fed Our Sea in Astounding Science Fiction (Aug-Sep, 1958)

3. Anderson, P. The Enemy Stars (Lippincott, Philadelphia, 1958)

4. Cameron, A. Astounding Science Fiction 62, 86-93 (1958).

5. Blaauw. A. Buil. astr. Inst. Neth. 15, 265-290 (1961)

6. Hubbard, W. B., MacFarlane, J. J., Anderson, J. D., Null, G. W. \& Biller, E. D. J. geophys. Res. 85, 5909-5916 (1980).

7. Ruppe, H. O. Introduction to Astronautics (Academic New York, 1966).

\section{Actin tracks}

SIR - Theriot and Mitchison ${ }^{1}$ have tracked actin in the lamella of moving fish epidermal keratocytes (FEK) using a new photoactivatable actin probe. They observed that the labelled actin filaments remained stationary with respect to the substratum in both locomoting and stationary cells. This interesting result may, however, represent the behaviour of only a subset of the actin microfilaments.

Centripetal motion of fluorescently labelled actin has been observed in nonlocomoting fibroblasts ${ }^{2}$ or growth cones $^{3}$. Theriot and Mitchison suggest that their failure to observe similar movement of actin could be due to an absence in keratocytes of control mechanisms present in fibroblasts and growth cones. But an alternative interpretation is to suppose that there are two populations of actin filaments in the lamella, a small stable population and a larger population undergoing rapid turnover. With photobleaching, a bleached spot would rapidly disappear because of turnover of the unstable filaments, and the stable actin would not be seen against the fluorescent background. With photoactivatable actin, however, one would see the opposite result: the unstable population would quickly fade while even a few stable filaments would remain visible against the dark background. The latter approach thus would tend to emphasize the most stable filaments, especially after longer time periods.

This hypothesis would reconcile some observations which we (D.F.K. and E.L.E.) have made with those in ref. 1. Previously we attempted to track the movements of rhodamine labelled actin filaments in the lamellae of locomoting FEK by observing a photobleached spot at room temperature ${ }^{2}$. We were unsuccessful, in part due to the short lifetime of the bleached spot (5-8 seconds). Using VEDIC microscopy, however, we have observed impressive cytoplasmic waves in stationary and locomoting FEK. There is good evidence that such waves reflect movement of actin ${ }^{3}$. As in nerve growth cones, the waves move at high velocity with respect to the substrate while the cell remains essentially stationary. The waves retract toward the nucleus upon addition of cytochalasin D, leaving the lamella essentially empty of actin filaments, except for a band at the leading edge and a few coarse filaments. Waves then reappear on washing out cytochalasin D (ref. 4). Our results thus suggest that at least a fraction of actin filaments may undergo constant centripetal flow in FEK.

We suspect that the stable actin filaments seen with the photoactivatable probe represent the ventral cortical 